\title{
Quantitative Estimation of Tabletability of Aceclofenac after Incorporation of Titanium Dioxide using Area under the Curve
}

\author{
Souvik Nandi, Satyaki Aparajit Mishra, Rudra Narayan Sahoo, Rakesh Swain, Subrata Mallick
}

School of Pharmaceutical Sciences, Siksha 'O' Anusandhan (Deemed to be University), Bhubaneswar, Odisha, INDIA.

\begin{abstract}
Background: Tablet manufacturing with direct compression is one of the leading industrial technique that consumes less time, labour and economic also. But the choice of excipients are critical in this case which will allow the drug to get compressed without granulation techniques. Purpose: Aceclofenac is a BCS class II non-steroidal anti-inflammatory drug, which exerts a low oral bioavailability because of low solubility in aqueous medium. The drug also suffers from compressibility and also shows poor tabletibility. Methods: We have attempted to improve tabletability by incorporating titanium dioxide $\left(\mathrm{TiO}_{2}\right)$ through kneading and solvent evaporation technique. Results: In the FTIR study revealed that NH and $\mathrm{Cl}$ aromatic stretching of aceclofenac has been affected significantly due to binding with $\mathrm{TiO}_{2}$. DSC thermogram ascertained the partial amorphization of the drug in the formulations. Evaluated tabletability from the area under the applied pressure vs tensile strength curve (AUTC) of A1T1 has shown a poor value in contrast to other formulations.
\end{abstract}

Key words: Aceclofenac, Tabletability, Titanium dioxide, Direct compression, Area under the curve.

\section{INTRODUCTION}

Tablet manufacturing with direct compression is one of the leading industrial techniques that consumes less time, labour and more economic also. But the choice of excipients are critical in the direct compression which allows the drug to get compressed without granulation techniques. The excipient must have symmetrical particle size distribution, must be compatible with the drug and other excipients and to bind a large amount of material while it is undergoing direct compression. Many research reports are available on directly compressible tablets but, quantitative estimation of tabletability using non-linear approach are very few in number.

Aceclofenac, a BCS class II non-steroidal anti-inflammatory drug possesses remarkable analgesic and antipyretic properties and used to treat rheumatoid and osteo arthritis. ${ }^{1-3}$ The drug also suffers from compressibility and shows poor tabletability. ${ }^{4,5}$
Talcum and magnesium stearate as the flow promoting agents even could not change in the flow properties of the drug in the presence of HPMC. The compression problem was observed in preparing aceclofenac mouth dissolving tablet by direct compression using microcrystalline cellulose and other excipients. Patil et al. 2012 has prepared aceclofenac agglomerates to improve its flowability and compressibility by emulsion solvent diffusion technique. ${ }^{6}$ The major limitations associated with the technique are the significant drug loss and polymer loss. ${ }^{7}$ Shete et al. 2018 have prepared cocrystals of Fenofibrate and Nicotinamide. In this study they have subjected the formulations to various tableting studies like hardness, thickness but they have not created any tabletability profile of those prepared tablets. ${ }^{8}$

This study deals with the tabletability behaviour of aceclofenac kneaded with $\mathrm{TiO}_{2}$ in different ratios by direct compression
Submission Date: 31-05-2019; Revision Date: 04-09-2019; Accepted Date: 22-11-2019

DOI: 10.5530/ijper.54.1.8 Correspondence:

Prof. Subrata Mallick, School of Pharmaceutical Sciences, Siksha 'O' Anusandhan (Deemed to be University), Bhubaneswar-751003, Odisha, INDIA. Phone: +91 06742386209 E-mail: profsmallick@gmail. com

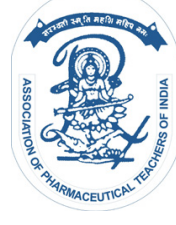

www.ijper.org 
technique. Quantitative estimation of tabletability has rarely been studied earlier for any tablet formulation by direct compression technique. Non-linear approach for estimation of tabletability quantitatively has been proposed in this work from simple relationship which is supposed to be more accurate relative to least square method. Titanium dioxide is having outstanding biocompatibility and the drug releasing performance can be tuned by designing formulation/medical implants. ${ }^{9,10}$

\section{MATERIALS AND METHODS Materials}

Titanium dioxide was purchased from Merck Specialities Pvt. Ltd., Mumbai, India. Microcrystalling cellulose was obtained from HIMEDIA Laboratories Pvt. Ltd., Mumbai, India. Aceclofenac pure drug was obtained as a gift sample from Mannequin Pharmaceuticals Pvt. Ltd., Bhubaneswar, Odisha.

\section{Preparation of Aceclofenac $\mathrm{TiO}_{2}$ Kneaded mixture}

Aceclofenac was dissolved in a minimal amount of acetone and a kneaded mixture was prepared with titanium dioxide at different ratios. ${ }^{11}$ The mass was dried at $50^{\circ} \mathrm{C}$ until constant weight and preserved in a desiccator.

\section{Characterization}

\section{FTIR Study}

FTIR study of pure drug and formulated powder sample was done using $\mathrm{KBr}$ pellet method. ${ }^{12}$ The FTIR spectrum was a mean of 80 times scan between the wave number of $400-4000 \mathrm{~cm}^{-1}$ in (Model: JASCO FTIR 4100type A). ${ }^{13}$

\section{Direct Compression}

The prepared formulation was mixed with microcrystalline cellulose by maintaining a ratio of 1:2. $200 \mathrm{mg}$ of the prepared mixture then subjected to the punching cavity manually. The pressure was varied while preparing the compacts by using the previously stated formulations. The pressures which have been used were 0.5,1, 1.5, 2 and 2.5 tons. ${ }^{14}$ The dimensions and hardness was then measured for determination of various physical and mechanical properties. ${ }^{8}$ Tensile strength was calculated by using formula: ${ }^{15}$

$$
T S=\frac{2 F}{\pi d h}
$$

Here, $\mathrm{F}$ is compression force applied in Newton force (unit: $\mathrm{kg} \cdot \mathrm{m} / \mathrm{S}^{2}$ ); $\mathrm{d}=$ diameter in meter, $\mathrm{h}=$ thickness of the tablet in meter.

\section{Kawakita model}

To relate between compaction pressure and volume reduction Kawakita and Ludde has proposed model as follows:

$$
\frac{P}{C}=\frac{P}{a}+\frac{1}{a b}
$$

In the above equation the 'P' signifies applied pressure, ' $\mathrm{a}$ ' and ' $\mathrm{b}$ ' both are constants which gives an idea of the maximum volume reduction and an inclination towards volume reduction respectively. ' $\mathrm{C}$ ' can be described as degree of volume reduction and can be calculated from

$$
C=1-\frac{V}{V_{0}}
$$

(where, $\mathrm{V}_{0}$ is the initial volume of the powder column and $\mathrm{V}$ is the volume under pressure). ${ }^{16,17}$

\section{RESULTS AND DISCUSSION}

\section{FTIR Study}

An intense peak was observed at $3317 \mathrm{~cm}^{-1}$ probably because of the amine group. ${ }^{18}$ (Figure 1) The peaks at 1715 and $1771 \mathrm{~cm}^{-1}$ may be formed due to stretching of two carbonyl $(\mathrm{C}=\mathrm{O})$ groups in the aceclofenac structure. ${ }^{19,20}$ The peak at $2969 \mathrm{~cm}^{-1}$ in both pure drug and formulations indicates the symmetric stretching of $\mathrm{CH}_{2}{ }^{21}$ In the formulations, the intensity of the peaks has decreased along with the increase of $\mathrm{TiO}_{2}$ concentration which indicated a possible binding of $\mathrm{TiO}_{2}$ and Acceclofenc.

\section{Direct Compression}

A model given by Sun et al. 2001 is mainly used in the case of expressing tabletability. The equation is as follows: ${ }^{22}$

$$
T S=k_{t} P+C
$$

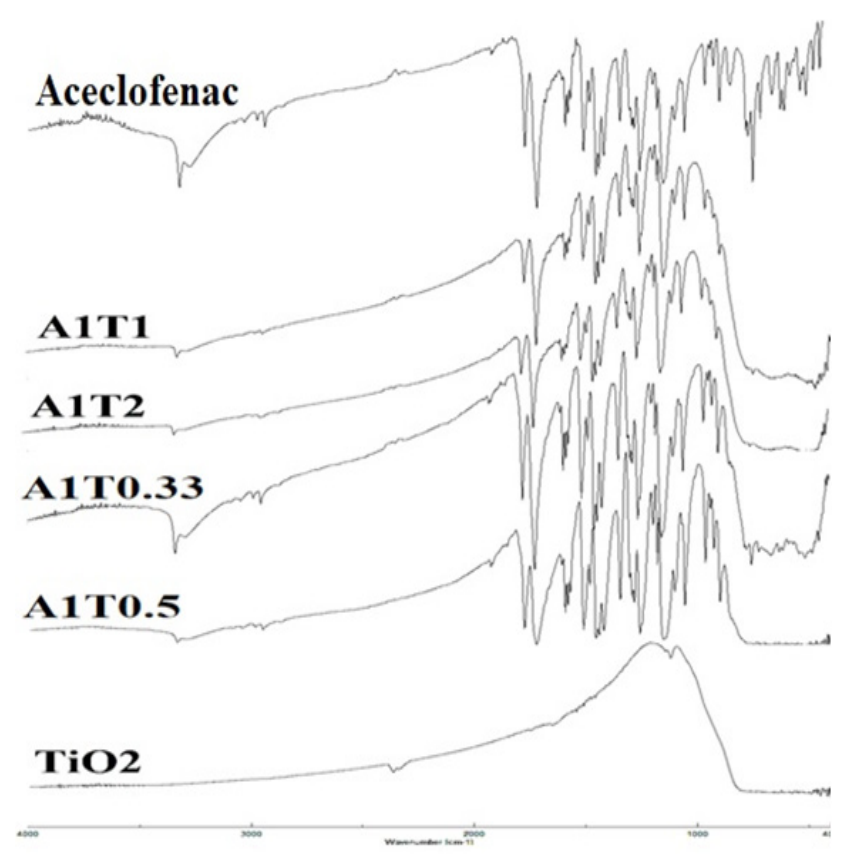

Figure 1: FTIR spectrum of aceclofenac and formulations 
Tabletibility of the composite powder material can be expressed by applied pressure vs tensile strength curve (Figure 2) where the slope value ' $k_{t}$ ' signifies

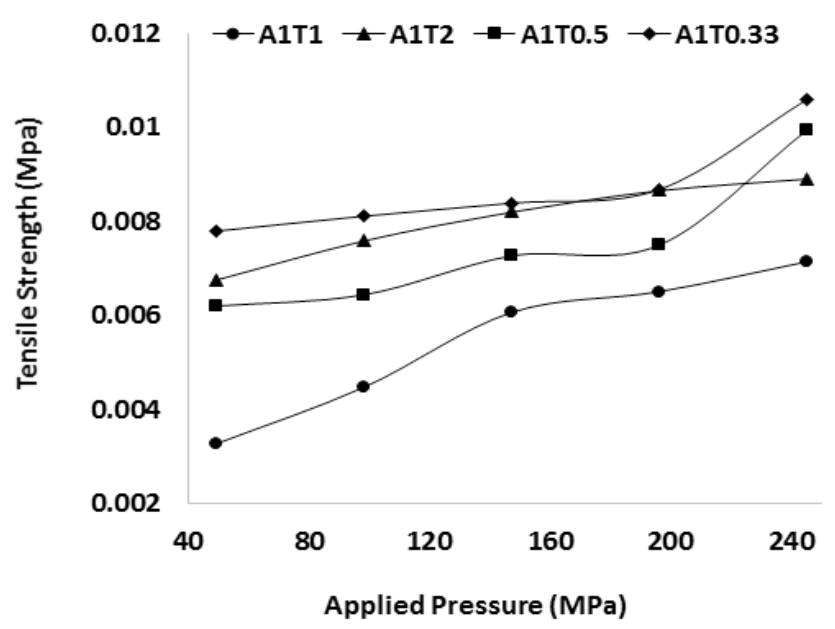

Figure 2: Tabletability profile of the formulations.

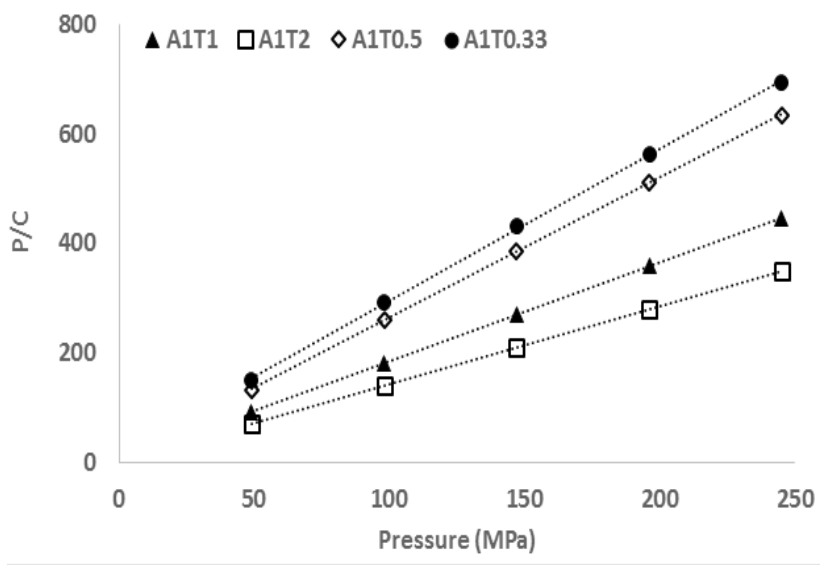

Applied Pressure (MPa)

Figure 3: Kawakita pressure Plot of the prepared formulations. tabletability coefficient. It can be quantified by Area under the applied pressure vs tensile strength curve (AUTC). (Table 1) The plot has clearly shown that the 1:1 formulation is poor in tabletibility in contrast to the other formulations. ${ }^{23}$

\section{Kawakita Model}

As per Kawakita linearity model, the highest compressibility (a) and inclination towards volume reduction (b) are seen with the formulation A1T2 (Figure 3) (Table 2).

\section{Proposed binding of Aceclofenac with $\mathrm{TiO}_{2}$}

Proposed binding between $\mathrm{TiO}_{2}$ and aceclofenac has been depicted in Figure 4a, b. Binding could be possible in either of the two ways. Titanium is getting attached to Chlorines while the amine group is getting attached with the oxygen present in $\mathrm{TiO}_{2}$. Chlorine may bind with the titanium by opposite charge attraction (4a) or formation of hydrogen bond between chlorine and titanium (4b).

\section{CONCLUSION}

Aceclofenac alone could not be tableted because of very poor compressibility by direct compression. Tabletability of A1T2, A1T0.5 and A1T0.33 formulations has been improved compared to A1T1. The kawakita parameters suggested that the inclination towards

\begin{tabular}{|c|c|c|}
\hline \multicolumn{3}{|c|}{ Table 1: Tabletability parameters. } \\
\hline Formulation Code & $\mathbf{K}_{\mathbf{t}}{ }^{*} \mathbf{1 0}^{\mathbf{6}}$ & AUTC \\
\hline A1T0.33 & 12.49 & 1.685 \\
\hline A1T0.5 & 17.32 & 1.435 \\
\hline A1T1 & 19.80 & 1.091 \\
\hline A1T2 & 10.94 & 1.584 \\
\hline
\end{tabular}

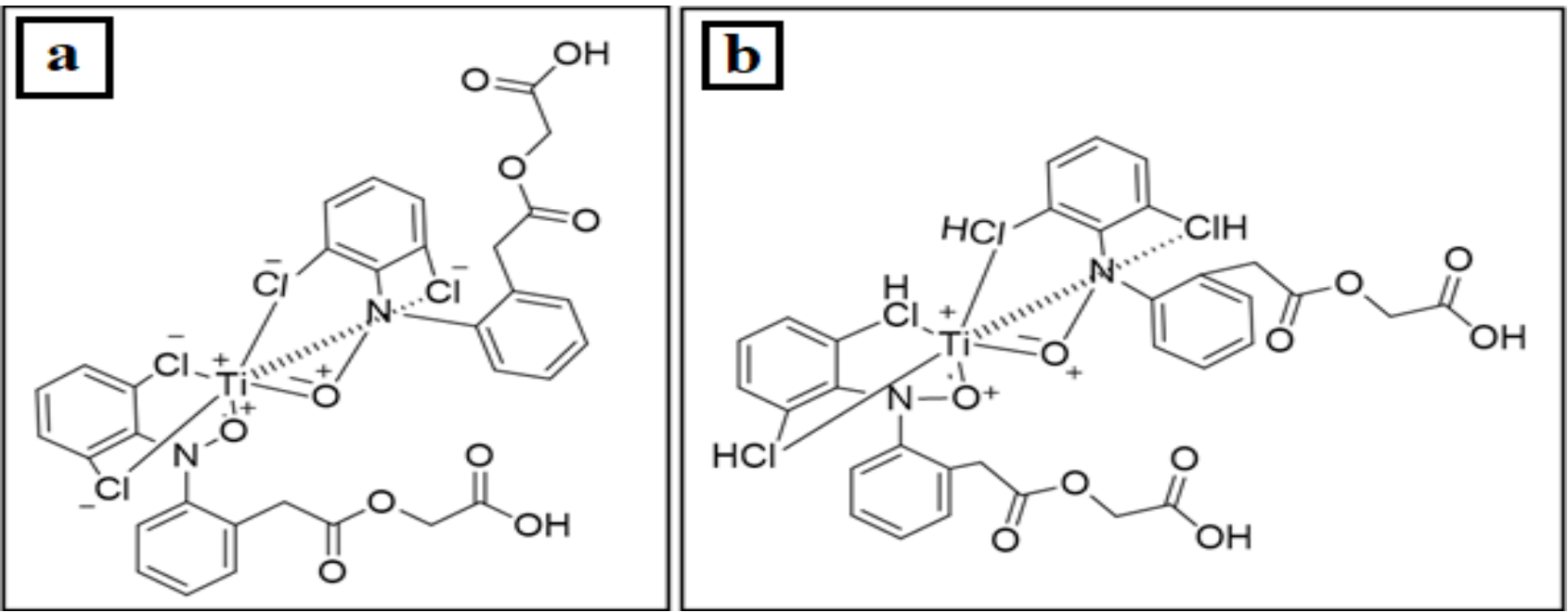

Figure 4: Proposed binding between $\mathrm{TiO}_{2}$ and Aceclofenac. Chlorine may bind with the titanium by opposite charge attraction (4a) or formation of hydrogen bond between chlorine and titanium (4b). 
Table 2. Kawakita Parameters of aceclofenac kneaded with $\mathrm{TiO}_{2}$ in different ratios

\begin{tabular}{|c|c|c|c|c|c|c|c|}
\hline Formulation Code & ${\text { Drug: } \mathrm{TiO}_{\mathbf{2}}(\mathbf{w} / \mathbf{w})}$ & Equation & $\mathbf{1 / a}$ & $\mathbf{1 / a b}$ & $\mathbf{a}$ & $\mathbf{a b}$ & $\mathbf{b}$ \\
\hline $\mathrm{A} 1 \mathrm{~T} 0.33$ & $1: 0.33$ & $\mathrm{y}=2.764 \mathrm{x}+19.17$ & 2.764 & 19.176 & 0.361 & 0.052 & 0.144 \\
\hline $\mathrm{A} 1 \mathrm{~T} 0.5$ & $1: 0.5$ & $\mathrm{y}=2.56 \mathrm{x}+8.16$ & 2.560 & 8.160 & 0.390 & 0.122 & 0.313 \\
\hline $\mathrm{A} 1 \mathrm{~T} 1$ & $1: 1$ & $\mathrm{y}=1.81 \mathrm{x}+3.14$ & 1.810 & 3.146 & 0.552 & 0.317 & 0.575 \\
\hline $\mathrm{A} 1 \mathrm{~T} 2$ & $1: 2$ & $\mathrm{y}=1.419 \mathrm{x}+1.26$ & 1.419 & 1.261 & 0.704 & 0.793 & 1.125 \\
\hline
\end{tabular}

volume reduction of the powder may be directly related to the amount of $\mathrm{TiO}_{2}$ incorporation.

\section{ACKNOWLEDGEMENT}

The authors are grateful to Dr. Monojranjan Nayak, President, Siksha 'O'Anusandhan University for financial support and laboratory facility. We are also grateful to receive Aceclofenac as gift sample from Mannequin Pharmaceuticals Pvt. Ltd., Bhubaneswar, Odisha.

\section{CONFLICT OF INTEREST}

The authors declare no conflict of interest.

\section{ABBREVIATIONS}

BCS: Biopharmaceutical Classification System; TiO Titanium Dioxide; DSC: Differential Scanning Calorimetry; FTIR: Fourier Transform Infrared Spectroscopy; AUTC: Area under the Tabletability Curve; HPMC: Hydroxypropylmethylcellulose; TS: Tensile Strength.

\section{REFERENCES}

1. Sethuraman N, Shanmuganathan S, Sandhya K, Anbarasan B. Design, Development and Characterization of Nano Structured Lipid Carrier for Topical Delivery of Aceclofenac. Indian J Pharm Educ. 2018;52(4):581-6.

2. Soni T, Nagda C, Gandhi T, Chotai NP. Development of Discriminating Method for Dissolution of Aceclofenac Marketed Formulations. Dissolut Technol. 2008;15(2):31-5.

3. Grau M, Guasch J, Montero JL, Felipe A, Carrasco E, Juliá S. Pharmacology of the Potent New Non-steroidal Anti-inflammatory Agent Aceclofenac. Arzneim-Forsch Drug Res. 1991;41(12):1265-76.

4. Mutalik S, Naha A, Usha AN, Ranjith AK, Musmade P, Manoj K, et al. Preparation, in vitro, Preclinical and Clinical Evaluations of Once Daily Sustained Release Tablets of Aceclofenac. Arch Pharm Res. 2007;30(2):22234.

\section{SUMMARY}

Aceclofenac is a poorly compressible drug and has a low tabletability. We have tried to overcome these drawbacks by incorporating $\mathrm{TiO}_{2}$ in the formulation. An increase in tablet ability has been observed after the addition of $\mathrm{TiO}_{2}$. Kawakita parameters also suggested that the inclination towards volume reduction of the powdered formulation is directly proportional to the amount of $\mathrm{TiO}_{2}$ added. 


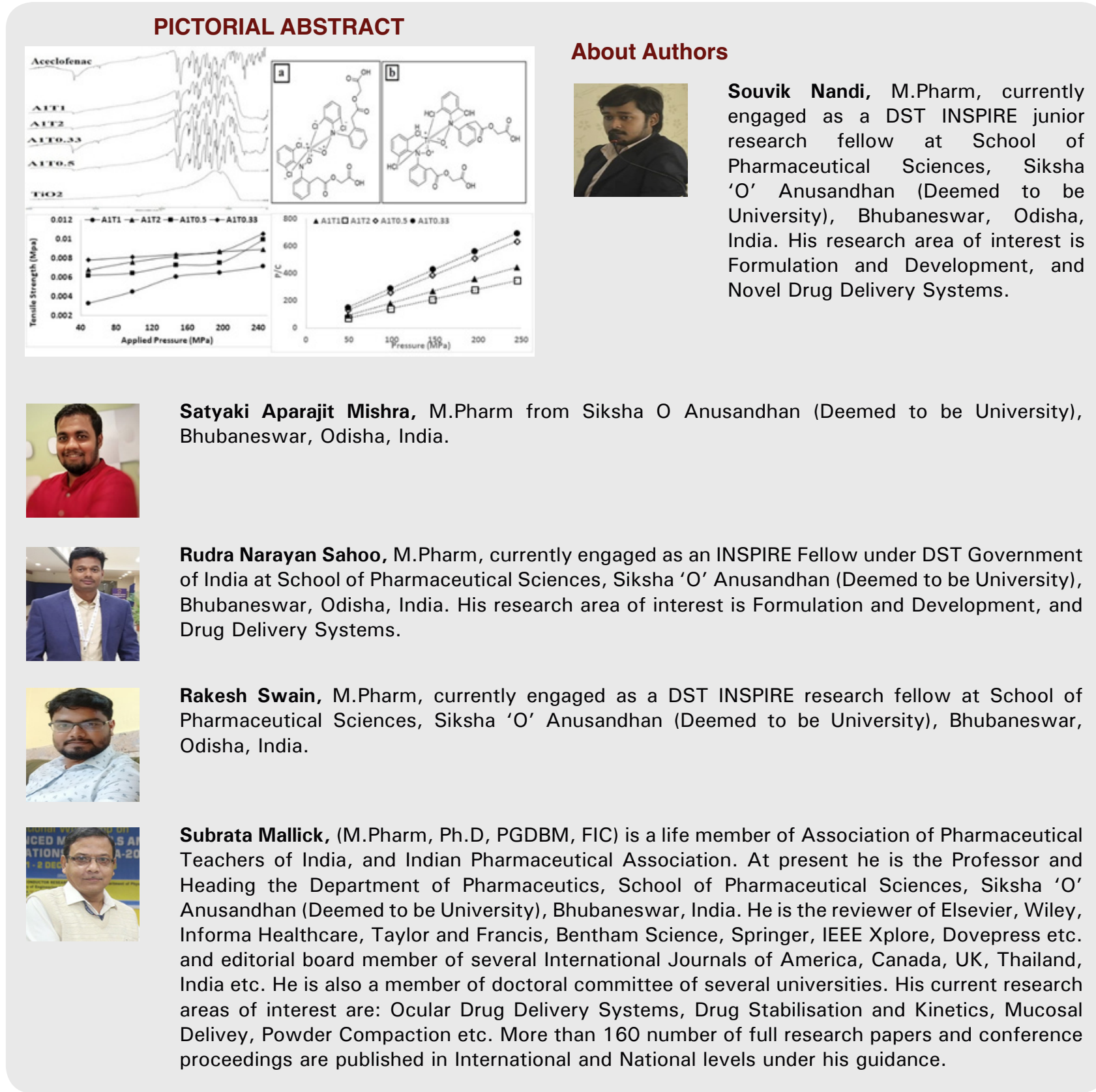

Cite this article: Nandi S, Mishra SA, Sahoo RN, Swain R, Mallick S. Quantitative Estimation of Tabletability of Aceclofenac after Incorporation of Titanium Dioxide using Area under the Curve. Indian $\mathrm{J}$ of Pharmaceutical Education and Research. 2020;54(1):68-72. 